Note. This article will be published in a forthcoming issue of the International Journal of Sports Physiology and Performance. The article appears here in its accepted, peer-reviewed form, as it was provided by the submitting author. It has not been copyedited, proofread, or formatted by the publisher.

Section: Original Investigation

Article Title: Effect of Cold on Proprioception and Cognitive Function in Elite Alpine Skiers

Authors: Sebastien Racinais ${ }^{1}$, Nadia Gaoua ${ }^{1,2}$, Khouloud Mtibaa ${ }^{3}$, Rodney Whiteley ${ }^{1}$, Christophe Hautier ${ }^{3}$, and Marine Alhammoud ${ }^{3,4}$

Affiliations: ${ }^{1}$ Aspetar Orthopaedic and Sports Medicine Hospital, Doha, Qatar. ${ }^{2}$ School of Applied Science, London South Bank University, UK. ${ }^{3}$ Center of Research and Innovation on Sport, University of Lyon 1, Villeurbanne, France. ${ }^{4}$ French Ski Federation, Annecy, France.

Journal: International Journal of Sports Physiology and Performance

Acceptance Date: March 28, 2016

(C2016 Human Kinetics, Inc.

DOI: $\underline{\text { http://dx.doi.org/10.1123/ijspp.2016-0002 }}$ 


\title{
Effect of Cold on Proprioception and Cognitive Function in Elite Alpine Skiers
}

Sebastien Racinais ${ }^{1}$, Nadia Gaoua ${ }^{1,2}$, Khouloud Mtibaa ${ }^{3}$, Rodney Whiteley ${ }^{1}$, Christophe Hautier ${ }^{3}$, Marine Alhammoud ${ }^{3,4}$

${ }^{1}$ Aspetar Orthopaedic and Sports Medicine Hospital, Doha, Qatar.

${ }^{2}$ School of Applied Science, London South Bank University, UK.

${ }^{3}$ Center of Research and Innovation on Sport, University of Lyon 1, Villeurbanne, France.

${ }^{4}$ French Ski Federation, Annecy, France.

Corresponding author: Sébastien Racinais, Athlete Health and Performance Research Centre, Aspetar Orthopaedic and Sports Medicine Hospital, PO Box 29222, Doha, Qatar. E-mail: sebastien.racinais@aspetar.com, Phone: +974.4413.2650, Fax: +974.4413.2020

Running Head: Cold and proprioception

Type: Original investigation

\author{
Abstract Word Count: 171 words \\ Text-Only Word Count: 3117 words \\ Number of Figures and Tables: 3 figures, 1 table
}


"Effect of Cold on Proprioception and Cognitive Function in Elite Alpine Skiers" by Racinais S et al.

International Journal of Sports Physiology and Performance

(C) 2016 Human Kinetics, Inc.

\section{ABSTRACT}

Purpose: To determine the effect of cold ambient conditions on proprioception and cognitive function in elite alpine skiers. Methods: 22 high-level Alpine skiers and 14 control participants performed a proprioceptive acuity (active movement extent discrimination) and a cognitive (planning task) test in cold $\left(8^{\circ} \mathrm{C}\right)$ and temperate $\left(24^{\circ} \mathrm{C}\right)$ ambient conditions. Results: All participants displayed an increase in thermal discomfort and in the amount of negative affects in cold environment (all $\mathrm{p}<0.05$ ). Average proprioceptive acuity was significantly better in the elite skiers $(0.46 \pm 0.12 \mathrm{deg})$ than in the control group $(0.55 \pm 0.12 \mathrm{deg})(\mathrm{p}<0.05)$ and was not affected by cold ambient conditions, except for a shift in the pattern of error (overestimation vs. underestimation, $\mathrm{p}<0.05)$. Cognitive performance was similar between elite skiers and control participants in temperate environments, but decreased in the cold in the control group only $(\mathrm{p}<0.05)$ becoming lower than in elite skiers $(\mathrm{p}<0.05)$. Conclusion: Elite alpine skiers showed a significantly better proprioceptive acuity than a control population and were also able to maintain their performance during a cognitive task in cold environment.

Keywords: Temperature; AMEDA; Ski; Exercise; Environment 
"Effect of Cold on Proprioception and Cognitive Function in Elite Alpine Skiers" by Racinais S et al.

International Journal of Sports Physiology and Performance

(C) 2016 Human Kinetics, Inc.

\section{INTRODUCTION}

Winter sports athletes as well as many other outdoor athletes are routinely exposed to cold during both training and competition. Whereas recreational alpine skiers wear well-insulated garments, competitive alpine skiers wear thin permeable racing suits. Elite alpine skiers are therefore exposed to cold on the chairlift or while waiting for the race start (i.e. skiers remove additional garments approximately 10 min before the start), with ambient temperatures during winter Olympics ranging from -5 to $+8^{\circ} \mathrm{C}[1]$ and even lower in many ski races. The risk of hypothermia and/or frostbite at these temperatures is generally considered minimal for most trained athletes due to high-metabolic rates (e.g. cross-country skiing) or brief exposures duration (e.g. alpine skiing) [1]. However, alpine skiing requires a variety of complex motor abilities including agility, coordination and proprioception [2] for which the effects of racing or waiting in a cold environment are not known.

In cold environments, an increase in shivering and muscle coactivation, and a decrease in muscle contraction velocity, nerve conduction velocity and stretch-reflex response [3], are likely to affect fine motor control, postural control, balance, and proprioception [4]. Of note, the effect of cold exposure on postural sway remained the same before or after cold habituation [4] suggesting that winter sports athlete are not exempt of such alterations. Importantly, proprioception has been shown to be an key component of performance [2] but also injury risk [5]. Proprioception corresponds to the ability to determine and coordinate body segment positions and movements in space [6]. It depends on neural pathways and cognitive programming [7]; both being potentially affected by cold environmental temperatures $[3,8]$.

To date, several studies have investigated the effect of therapeutic cryotherapy (i.e. localized cooling) on various balance and proprioception tests, but these studies have reported 
"Effect of Cold on Proprioception and Cognitive Function in Elite Alpine Skiers" by Racinais S et al.

International Journal of Sports Physiology and Performance

(C) 2016 Human Kinetics, Inc.

conflicting results [9] depending on the cooling methods and exposed area. For example, the Star

Excursion Balance Test is impaired when cooling the entire leg (i.e. from the foot up to the hip), but not when cooling only the ankle or the lower leg [10]. The effect of cold ambient conditions on winter sports athletes remain to be investigated during whole body cold exposure rather than during or after localized segmental cooling. In addition, even if cold acclimatization is minimal in humans [1], repeated cold-exposures induce psychological adaptations such as an increase in thermal comfort [11] and elite alpine skiers might therefore display different responses than the general population.

The aim of this study was to determine the effect of cold ambient conditions on proprioception and cognitive function in elite alpine skiers. We hypothesized that cold exposure would alter both active movement-based proprioception and cognitive function.

\section{METHODS}

\section{Participants}

Twenty-two high-level alpine skiers (179 $\pm 4 \mathrm{~cm}, 83 \pm 6 \mathrm{~kg}, 26 \pm 4 \mathrm{yrs})$ and 14 control participants (178 $\pm 5 \mathrm{~cm}, 78 \pm 5 \mathrm{~kg}, 33 \pm 6$ yrs) participated in this study after providing their written informed consent. The skiers were male Caucasian, part of the French Alpine national ski team (6 Europa Cup skiers and 16 World Cup skiers, including several Olympic medalists and world champions) and were accustomed to training and competing in cold environments for approximately 200 days a year for several years. The control participants were male Caucasian, recreational athletes (competitor or fitness instructor, with a ski level ranging from beginner to intermediate) who were living and training in a warm environment (i.e. Middle East). No participants were under medication except one of the skiers taking NSAID. The protocol conformed to the recommendations of the Declaration of Helsinki. The project was approved by 
"Effect of Cold on Proprioception and Cognitive Function in Elite Alpine Skiers" by Racinais S et al.

International Journal of Sports Physiology and Performance

(C) 2016 Human Kinetics, Inc.

Aspetar scientific committee (approval number CMO/000058/fj) and by ADL-Q ethics committee (approval number E20140000011).

\section{General procedure}

Following a familiarization session, the participants underwent two additional experimental sessions in thermoneutral (Control, $\mathrm{CON}, 24^{\circ} \mathrm{C}$ ) and cold $\left(\mathrm{COLD}, 8^{\circ} \mathrm{C}\right.$ ) environments, in a counterbalanced order, wearing shorts and t-shirts in the two sessions. Each of the experimental sessions included a rest period $(10 \mathrm{~min})$ followed by a proprioception test $(\sim 10$ min) and a cognitive test ( $\sim 10 \mathrm{~min})$, in randomized order between participants.

\section{Physiological and Subjective responses}

Environmental temperature was maintained constant inside an environmental chamber (Tescor, Warminster, USA). Skin temperatures were recorded over the chest (under the t-shirt), hand and calf (above the ski boot) using skin thermistors (precision $0.1^{\circ} \mathrm{C}$, range -1.0 to $+50.0^{\circ} \mathrm{C}$, DM 852, Ellab, Denmark) in order to calculate the mean skin temperature based on the Burton [12] formula as: 0.5 chest +0.14 hand +0.36 calf temperatures. Due to ski boots tightness, no temperature sensors were placed at the ankle level to avoid affecting the proprioception test.

Measures for thermal sensation and thermal discomfort were recorded at the beginning and end of the test using visual analogic scales ranging from very cold to very hot (blue to red scale) and from very comfortable to very uncomfortable (white to black graduation scale), respectively. Corresponding scores ranging from 0 (very cold / very comfortable) to 20 (very hot / very uncomfortable) were on the reverse side of both scales and visible only to the researcher.

Before starting the cognitive assessment the subjects completed the Positive and Negative Affect Schedule (PANAS) [13]. The PANAS is a 20-item self-report psychometric scale developed to measure the largely independent constructs of positive (PA) and negative (NA) affects as both 
states and traits [13]. PANAS has a high internal consistency and displays good test-retest reliability across different time frames, as well as good external validity for general psychological stress and depressed affect [13]. High-NA reflects subjective distress and unpleasurable engagement, and low NA the absence of these feelings. By contrast, PA represents the extent to which an individual experiences pleasurable engagement with the environment. The positive and negative affects scores were computed and the negative/positive affect ratio was calculated.

\section{Proprioception test}

Proprioceptive acuity was assessed as the ability to discriminate between movements using an Active Movement Extent Discrimination Apparatus (AMEDA, Fig. 1) [14]. Participants were wearing ski boots fixed to ski bindings. The left binding was attached to a fixed support and the right binding was attached to a mobile support. The mobile support could freely move until a fixed stop set at 5 different eversion angles $(10.5,11.2,11.9,12.6$ and $13.3 \mathrm{deg})$ in a random order. Participants had to actively evert the foot and recognize the angle allowed by pressing a keypad numbered from 1 to 5 . Following 3 practice trials at each angle, participants underwent a total of 50 trials (10 at each angle, random order). Participants were facing a white wall and could not see the apparatus. High Intraclass Correlation Coefficients (ICC) of 0.85 and 0.89 [15] have been previously reported for such active movement discrimination procedures [16]. The average absolute error was computed in degree. The number of overestimations (i.e. an estimated eversion deeper than the real one) and underestimations (i.e. an estimated eversion smaller than the real one) was also recorded.

\section{Cognitive evaluation}

Participants were seated in an upright position in front of a desk and performed a planning task (OTS: One Touch Stockings of Cambridge) using Cantab software (CANTABeclipse, 
Cambridge Cognition, Cambridge, UK) and hardware (tactile screen). Participants were shown two horizontal displays containing three colored balls. The displays were presented in such a way that they could be perceived as stacks of colored balls held in stockings suspended from a beam. Participants were required to mentally solve the minimum number of moves required to reproduce on the lower display the same pattern than on the upper display. Participants were then required to indicate the correct solution at the bottom of the screen by selecting a number from 1 to 7 . In case of error, the test continued until finding the correct response. Participants were instructed to firstly prioritize the accuracy of the response and secondly to respond as fast as possible as soon as they knew the answer. The outcome measure was the number of problems solved on the first choice (i.e. accuracy), the latency to first response and the latency to correct response. Measures were analyzed for two different levels of complexity requiring four (OTS-4) and six moves (OTS-6). Each measure was obtained by averaging the score obtained over four trials. This test depends on frontal lobe functioning and is sensitive to the environmental conditions [17].

\section{Statistical analysis}

Data were coded in SPSS 20 (SPSS, Chicago, IL, US). Data were analyzed using a two-way ANOVA with 2 groups (ski versus control, between subjects) x 2 conditions (temperate versus cold, within subjects). Least Significant Difference (LSD) pairwise comparisons were used to compare the effect of condition at each time interval in case of significant condition $\mathrm{x}$ task interaction. The level of statistical significance was set at $\mathrm{p}<0.05$. Effect-sizes are described in terms of partial eta-squared $(\eta 2$; with $\eta 2 \geq 0.06$ representing a moderate difference and $\eta 2 \geq 0.14$ a large difference). 
"Effect of Cold on Proprioception and Cognitive Function in Elite Alpine Skiers" by Racinais S et al.

International Journal of Sports Physiology and Performance

(C) 2016 Human Kinetics, Inc.

\section{RESULTS}

\section{Physiological and Subjective responses}

Skin temperature and thermal sensation were lower and thermal discomfort was higher in cold than temperate ambient conditions (all $\mathrm{p}<0.001, \eta 2>0.57$; Table 1 ). In addition, the amount of negative affects $(p=0.003, \eta 2=0.23)$, but not positive affects $(p=0.286, \eta 2=0.03)$, was higher in cold than temperate ambient conditions, leading to a higher negative/positive affects ratio in the cold $(\mathrm{p}<0.001, \eta 2=0.32 ;$ Table 1$)$.

Neither temperatures, thermal sensation nor thermal discomfort were significantly different between groups or displayed an interaction effect (all $\mathrm{p}>0.116, \eta 2<0.05$; Table 1). Interestingly, the elite skiers participating to this study reported more pronounced positive $(p=0.020, \eta 2=0.15)$ and negative $(\mathrm{p}=0.049, \eta 2=0.11)$ affects than the control group (Table 1$)$. This overall effect was independent of the condition (both $\mathrm{p}>0.16, \eta 2<0.06$ ) and the ratio negative/positive affects was identical between the groups $(\mathrm{p}=0.952, \eta 2<0.01)$.

\section{Proprioceptive acuity}

The pattern of response to the AMEDA test is displayed in Fig. 1. The average error was significantly lower in the elite skiers than in the control group $(\mathrm{p}=0.010, \eta 2=0.18$; Fig. 1$)$ but was not different in cold and temperate ambient conditions $(\mathrm{p}=0.31, \eta 2=0.03)$. However, whereas the errors were predominantly due to underestimation in temperate ambient conditions, this pattern changed in cold ambient conditions $(\mathrm{p}=0.010, \eta 2=0.18$; Fig. 1$)$.

\section{Cognitive evaluation}

There was a significant interaction effect between group and conditions for both the simple $(\mathrm{OTS}-4 ; \mathrm{p}=0.017, \eta 2=0.16)$ and complex (OTS-6; $\mathrm{p}=0.007, \eta 2=0.20)$ cognitive task. Cognitive performances were similar between the elite skiers and the control population in temperate 
environment (OTS-4 p=0.482; OTS- $6 \mathrm{p}=0.727$ ). However, accuracy decreased with cold exposure in the control group only (OTS-4 $\mathrm{p}=0.026$; OTS- $6 \mathrm{p}=0.015$ ) becoming lower than in the elite skiers (OTS-4 p=0.003; OTS-6 p=0.007) (Fig. 2).

Of note, the elite athletes participating to this study tend to take more time before answering than the control group (OTS-4 $\mathrm{p}=0.083, \eta 2=0.09$; OTS $-6 \mathrm{p}=0.056, \eta 2 \geq 0.11$ ). This difference is becoming significant when performing the complex task (OTS-6) in cold environment where, in parallel of a better accuracy, the skiers took significantly more time before answering $(\mathrm{p}=0.046)$ and before finding the correct answer ( $\mathrm{p}=0.013$ ) than the control participants (Fig. 3).

\section{DISCUSSION}

The aim of this study was to determine the effect of cold ambient conditions on proprioception and cognitive function in elite alpine skiers. Our data showed that i) elite skiers had a significantly better active proprioceptive acuity than control participants, ii) the pattern of error during the AMEDA test was different in cold and temperate ambient conditions but the average error remained similar, and iii) a cold environment impaired accuracy during a cognitive task in the control participants but not in the elite skiers. These data partly validate the original hypothesis that active movement-based proprioception and cognitive function will be impaired in cold environment. However, the impairment in proprioceptive acuity does not affect the average error and elite skiers were able to maintain their level of accuracy during the cognitive task in cold ambient conditions.

\section{Elite skiers display a better proprioceptive acuity}

There is an equipoise in the literature regarding the effect of exercise training on proprioception [18]. Some studies have reported an improvement in knee proprioception after training $[19,20,21]$ but sometimes without concomitant improvement in the foot / ankle complex 
[21]. Moreover, there was no improvement in ankle position sense after 5-month of ballet training [22]. Proprioception scores for the ankle, knee, shoulder, spine, or fingers have been shown to be associated with performance level achieved but did not correlate with the number of years of sportspecific training [23]. The only study investigating ski expertise reported that skiers of national level had a similar postural control than skiers of regional level with ski boots but displayed an impaired postural stability without ski boots speculatively in relation with a long term effect of repeatedly wearing ski boots [24].The current data add to the debate that elite skiers have a better active proprioceptive acuity than the general population during an eversion task reproducing their sporting activity with ski boots (Fig. 1).

This better proprioceptive acuity is only one of the aspects characterizing elite Alpine skiers and the current results do not allow to discriminate if elite skiers acquired this better proprioception through their training or have an inherent better proprioception as part of their skills that led them to reach this level of ski expertise. Given that skiing is performed in a cold environment we hypothesized that, irrespective of being acquired or inherent, the better proprioception of the elite skiers would be more marked in a cold environment. The various methodology and cold treatment (mostly localized cryotherapy) used in the literature failed to unequivocally characterize the effect of cold on proprioception [9]. The current study does not allow concluding on the effect of a localized aggressive cooling on proprioception but our data suggest that the average active proprioceptive acuity is not different in cold and temperate environments, even when using a test sensitive enough to differentiate ski expertise. Of note, despite similar average proprioceptive acuity errors in cold and temperate environments, cold exposure modified the pattern of error (Fig. 1). Speculatively, we could suggest that this may be a flawed response likely to modify the perception of the skier and therefore the required accurate ski 
"Effect of Cold on Proprioception and Cognitive Function in Elite Alpine Skiers" by Racinais S et al.

International Journal of Sports Physiology and Performance

(C) 2016 Human Kinetics, Inc.

placement to safely execute turning and other skiing maneuvers. In any case, the effects of cold ambient conditions on the proprioception test were similar for the skiers and the control participants supporting previous data showing that the effect of cold exposure on postural sway remained the same before or after cold habituation [4].

\section{Cold exposure affects cognitive function}

The current data showed a decrease in cognitive performance (planning task) with cold exposure in the control group (Fig. 2). This observation confirms that cold environmental temperatures can significantly affect cognitive performance [8] including concentration, vigilance, memory, and reasoning [25]. Of note, whereas hot ambient conditions have previously been reported to impair complex (OTS-6) but not simple (OTS-4) planning tasks [26], the impairment in cold ambient conditions observed in the current study was similar for both tasks. It has been suggested that these impairments in cognitive performance might be related to a decrease in available resources due to the distraction caused by cold discomfort that could consume central attention resources [27]. This mechanism is supported, at least for the control group, by the current data showing an increase in thermal discomfort with cold exposure (Table 1).

However, despite similar levels of discomfort, the elite skiers were able to maintain their cognitive function in the cold. Even if the current data failed to show significant differences in thermal sensation and discomfort responses between the control group and the elite skiers, previous studies showed that cold adaptation/habituation can improve thermal sensation and comfort [11]. In the absence of a 'cold habituation' intervention, we can only speculate that their usual training in cold environments may have made it possible for elite skiers to maintain their attention focused on the task. Importantly, this occurred at the expense of higher latency to response and a longer duration to find the correct answer during OTS-6 (Fig. 3). In accordance 
with Mäkinen [28] and following the arousal theory, the skiers became more aroused or engaged in performing the task given that they perceived the cold as a challenge and consequently devoted greater attention (and time) in completing the task. Conversely, the control group viewed it as a too stressful situation and could not, or did not want to, increase the time allocated to the task in the cold.

This model is supported by the PANAS results from the current experiment showing that the skiers exhibited more intense positive and negative responses to the PANAS. This could be related to the fact that athletes exhibit higher emotional intelligence [29] making them more aware of their own emotions. By better recognizing their positive and negative affects, skiers might be able to trigger the appropriate responses and coping strategies in difficult situations [30].

\section{Perspectives}

Proprioception has been recognized as an important factor for both injury risk [5] and the recovery of athletic performance [31] and is of particular importance in alpine skiing [2]. Consequently, it is now highly recommended to include a proprioceptive component in sports medicine practice, training as well as rehabilitation programs [18]. In order to maximize the ecological validity of the proprioception testing, the participants of the current study were tested in weight-bearing situation, wearing ski boots and actively moving the ski bindings at their own pace. Active movements made by the subject are discrete, self-initiated and result in a functional interaction with the environment. However, other environmental variables (e.g. visibility, change in snow surface conditions) remain to be investigated to characterize the environmental factors affecting injury risk and performance.

The current protocol included a passive cold exposure similar to the conditions and duration experienced by the skiers on the chairlift during training [32] or before the start in 
competition. Indeed, skiers remove their pants and jacket approximately 10 min before the start of the race and wear only a thin one-piece racing suit over a single under-layer while standing in the cold. Racing suits must have a minimum permeability of $30 \mathrm{~L} / \mathrm{m}^{2} / \mathrm{sec}$ (The alpine international ski competition rules, version of July 2015) and therefore provide limited cold protection. In the current study, in order to allow for comparison with a control group, the skiers did not wear a racing suit and the temperature was therefore set at the high-end of the ambient temperatures reported during winter Olympics $\left(-5\right.$ to $\left.+8^{\circ} \mathrm{C}\right)$ [1]. Further studies are required to estimate the impact of colder environment.

\section{Conclusion}

The current data showed a significantly better proprioceptive acuity in elite alpine skiers than a control population. This difference remained across environmental conditions. Proprioception was not markedly altered in cold ambient conditions, other than a shift in the pattern of error. Moreover, despite more errors during the planning task performed in cold than temperate environments in the control group, elite alpine skiers were able to maintain their level of cognitive performance.

\section{Practical implications}

- Elite skiers have a better proprioceptive acuity of the ankle eversion in ski boots than a control population.

- Cognitive functions are altered in a cold environment in control participants but not in elite skiers. 
"Effect of Cold on Proprioception and Cognitive Function in Elite Alpine Skiers" by Racinais S et al.

International Journal of Sports Physiology and Performance

(C) 2016 Human Kinetics, Inc.

\section{Acknowledgement:}

The authors thank Nicolas Cabaret and Thibaut Trameau for their role in the study implementation.

The authors thank Professor Gordon Waddington for his support with the proprioception assessment. The authors thank the skiers, the medical team and the coaching team from the French Ski Federation for their effort. Funding for the conduct of this study was provided by Aspetar Orthopaedic and Sports Medicine Hospital. The funders had no role in study design, data collection and analysis, decision to publish, or preparation of the manuscript. The authors have no conflicts of interest that are directly relevant to the content of this manuscript. 
"Effect of Cold on Proprioception and Cognitive Function in Elite Alpine Skiers" by Racinais S et al.

International Journal of Sports Physiology and Performance

(C) 2016 Human Kinetics, Inc.

\section{REFERENCES}

1 Bergeron MF, Bahr R, Bärtsch P, et al. International Olympic Committee consensus statement on thermoregulatory and altitude challenges for high-level athletes. British Journal of Sports Medicine 2012;46:770-9.

2 Malliou P, Amoutzas K, Theodosiou A, et al. Proprioceptive training for learning downhill skiing. Percept Mot Skills 2004;99:149-54.

3 Racinais S, Oksa J. Temperature and neuromuscular function. Scand J Med Sci Sports 2010;20 Suppl 3:1-18.

4 Mäkinen TM, Rintamäki H, Korpelainen JT, et al. Postural sway during single and repeated cold exposures. Aviat Space Environ Med 2005;76:947-53.

5 Lauersen JB, Bertelsen DM, Andersen LB. The effectiveness of exercise interventions to prevent sports injuries: a systematic review and meta-analysis of randomised controlled trials. British Journal of Sports Medicine 2014;48:871-7.

6 Goble DJ. Proprioceptive Acuity Assessment Via Joint Position Matching: From Basic Science to General Practice. Phys Ther 2010;90:1176-84.

7 Lephart SM, Pincivero DM, Giraldo JL, et al. The role of proprioception in the management and rehabilitation of athletic injuries. The American Journal of Sports Medicine 1997;25:130-7.

8 Palinkas LA, Reed HL, Reedy KR, et al. Circannual pattern of hypothalamic-pituitarythyroid (HPT) function and mood during extended antarctic residence.

Psychoneuroendocrinology 2001;26:421-31.

9 Furmanek MP, Słomka K, Juras G. The effects of cryotherapy on proprioception system. BioMed Research International 2014;2014:696397.

10 Montgomery RE, Hartley GL, Tyler CJ, et al. Effect of segmental, localized lower limb cooling on dynamic balance. Med Sci Sports Exerc 2015;47:66-73.

11 Young AJ. Homeostatic Responses to Prolonged Cold Exposure: Human Cold Acclimatization. In: Fregly MJ, Blatters CM, eds. Handbook of Physiology. Environmental Physiology. New York: : John Wiley \& Sons, Inc. 1996. 419-38.

12 Burton AC. The Application of the Theory of Heat Flow to the Study of Energy Metabolism Five Figures. J Nutr 1934;:597-533.

13 Watson D, Clark LA, Tellegen A. Development and validation of brief measures of positive and negative affect: the PANAS scales. J Pers Soc Psychol 1988;54:1063-70.

14 Waddington GS, Shepherd RB. Ankle injury in sports: Role of motor control systems and implications for prevention and rehabilitation. Physical Therapy Reviews 1996;1:79-87. 
"Effect of Cold on Proprioception and Cognitive Function in Elite Alpine Skiers" by Racinais S et al.

International Journal of Sports Physiology and Performance

(C) 2016 Human Kinetics, Inc.

15 Fleiss JL. The Design and Analysis of Clinical Experiments. New York: : WileyInterscience 1986.

16 Han J, Waddington G, Anson J. A novel device for the measurement of functional finger pinch movement discrimination. Applied Mechanics and Materials 2011;66-68:620-5.

17 Gaoua N, Grantham J, Massioui El F, et al. Cognitive decrements do not follow neuromuscular alterations during passive heat exposure. Int J Hyperthermia 2011;27:109.

18 Ashton-Miller JA, Wojtys EM, Huston LJ. Can proprioception really be improved by exercises? Knee surgery 2001;9:128-36.

19 Daneshjoo A, Mokhtar AH, Rahnama N, et al. The effects of comprehensive warm-up programs on proprioception, static and dynamic balance on male soccer players. PLoS ONE 2012;7:e51568.

20 Waddington G, Seward H, Wrigley T, et al. Comparing wobble board and jump-landing training effects on knee and ankle movement discrimination. J Sci Med Sport 2000;3:449-59.

$21 \mathrm{Xu}$ D, Hong Y, Li J, et al. Effect of tai chi exercise on proprioception of ankle and knee joints in old people. British Journal of Sports Medicine 2004;38:50-4.

22 Schmitt H, Kuni B, Sabo D. Influence of professional dance training on peak torque and proprioception at the ankle. Clin J Sport Med 2005;15:331-9.

23 Han J, Waddington G, Anson J, et al. Level of competitive success achieved by elite athletes and multi-joint proprioceptive ability. J Sci Med Sport 2015;18:77-81.

24 Noe F, Paillard T. Is postural control affected by expertise in alpine skiing? British Journal of Sports Medicine 2005;39:835-7.

25 Hoffman RG. Human psychological performance in cold environments. In: Pandolf KB, Burr RE, eds. Textbook of Military Medicine. Medical Aspects of Harsh Environments. Washington DC: : Medical Aspects of Harsh Environments 2001. 384-410.

26 Gaoua N, Racinais S, Grantham J, et al. Alterations in cognitive performance during passive hyperthermia are task dependent. Int J Hyperthermia 2011;27:1-9.

27 Davis FM, Baddeley AD, Hancock TR. Diver performance: the effect of cold. Undersea Biomed Res 1975;2:195-213.

28 Mäkinen T, PALINKAS L, REEVES D, et al. Effect of repeated exposures to cold on cognitive performance in humans. Physiol Behav 2006;87:166-76.

29 Costarelli V, Stamou D. Emotional intelligence, body image and disordered eating attitudes in combat sport athletes. Journal of Exercise Science \& Fitness 2009;7:104-11. 
30 Scott VB, Robare RD, Raines DB, et al. Emotive writing moderates the relationship between mood awareness and athletic performance in collegiate tennis players. North American Journal of Psychology 2003;5:311-24.

31 Katayama M, Higuchi H, Kimura M, et al. Proprioception and performance after anterior cruciate ligament rupture. International Orthopaedics (SICOT) 2004;28:278-81.

32 Suzuki E, Ohya T, Ito R, et al. Physiological responses in Alpine skiers during on-snow training simulation in the cold. Int J Sports Med 2014;35:392-8. 

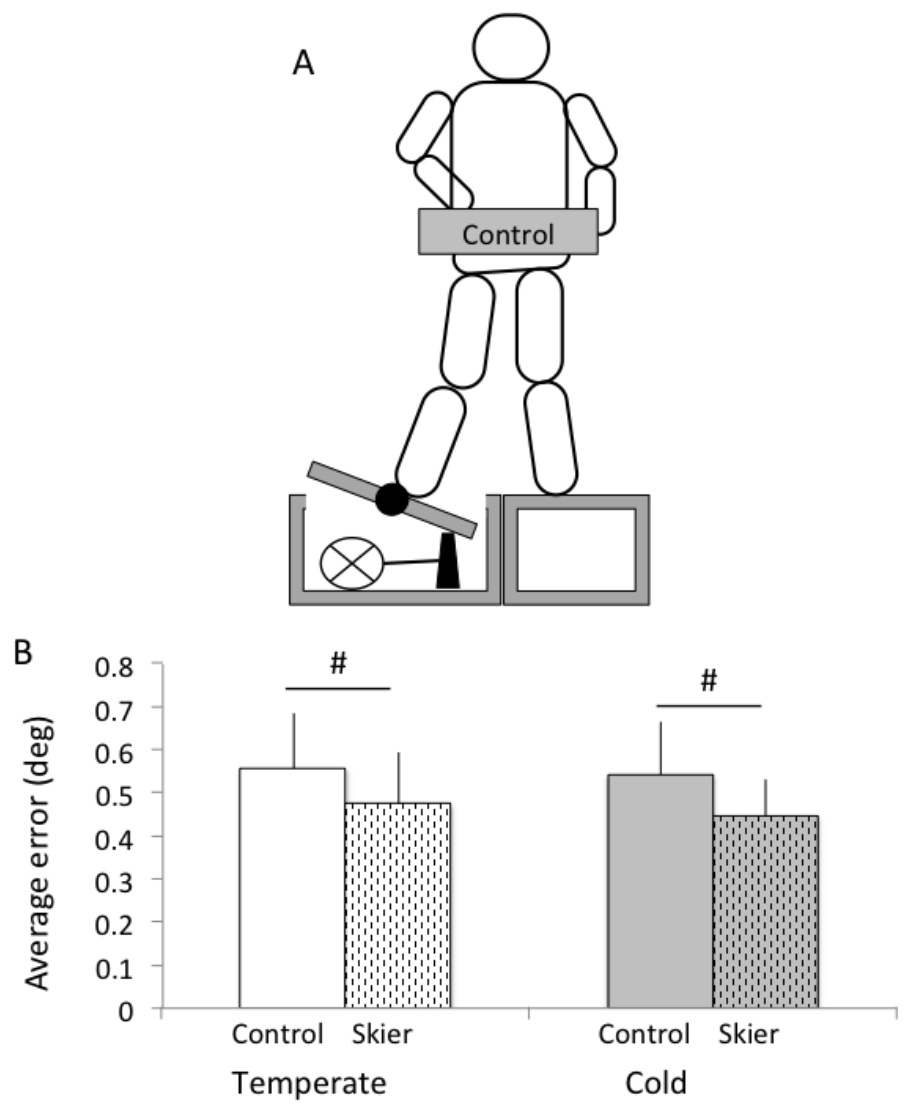

C Number of Overestimation Number of Underestimation

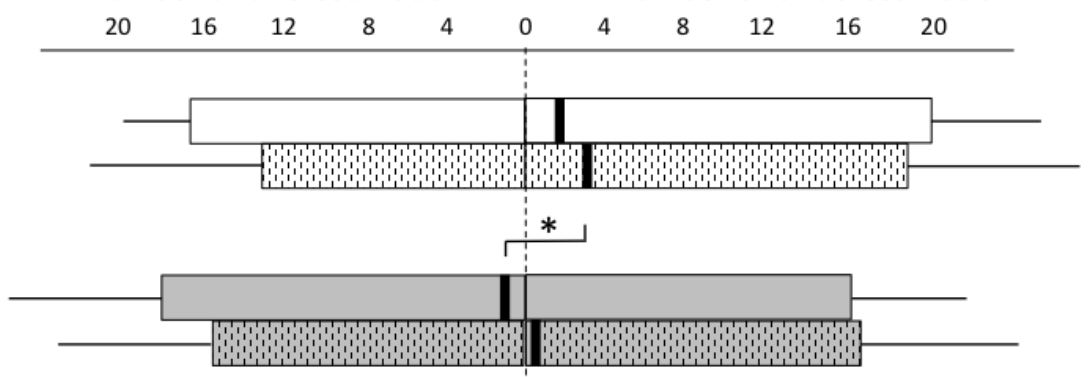

Figure 1. Proprioceptive acuity estimated by the Active Movement Extent Discrimination Apparatus (AMEDA). Participants were equipped with ski boots fixed to ski bindings (A). The right binding was attached to a mobile support moving freely until a fixed stop randomly set at 5 different eversion angles by an engine. The average absolute error (B) and the numbers (C) of overestimation (i.e. an estimated eversion deeper than the real one) and underestimation (i.e. an estimated eversion smaller than the real one) were computed from 50 trials in cold $\left(8^{\circ} \mathrm{C}\right.$, grey bars) and temperate $\left(24^{\circ} \mathrm{C}\right.$, white bars) environments in elite skiers (dashed bars) and control participants (plain bars). \# difference between groups, ${ }^{*}$ difference between condition, $\mathrm{p}<0.05$. Value in mean $\pm \mathrm{SD}$. 
"Effect of Cold on Proprioception and Cognitive Function in Elite Alpine Skiers" by Racinais S et al. International Journal of Sports Physiology and Performance

(C) 2016 Human Kinetics, Inc.
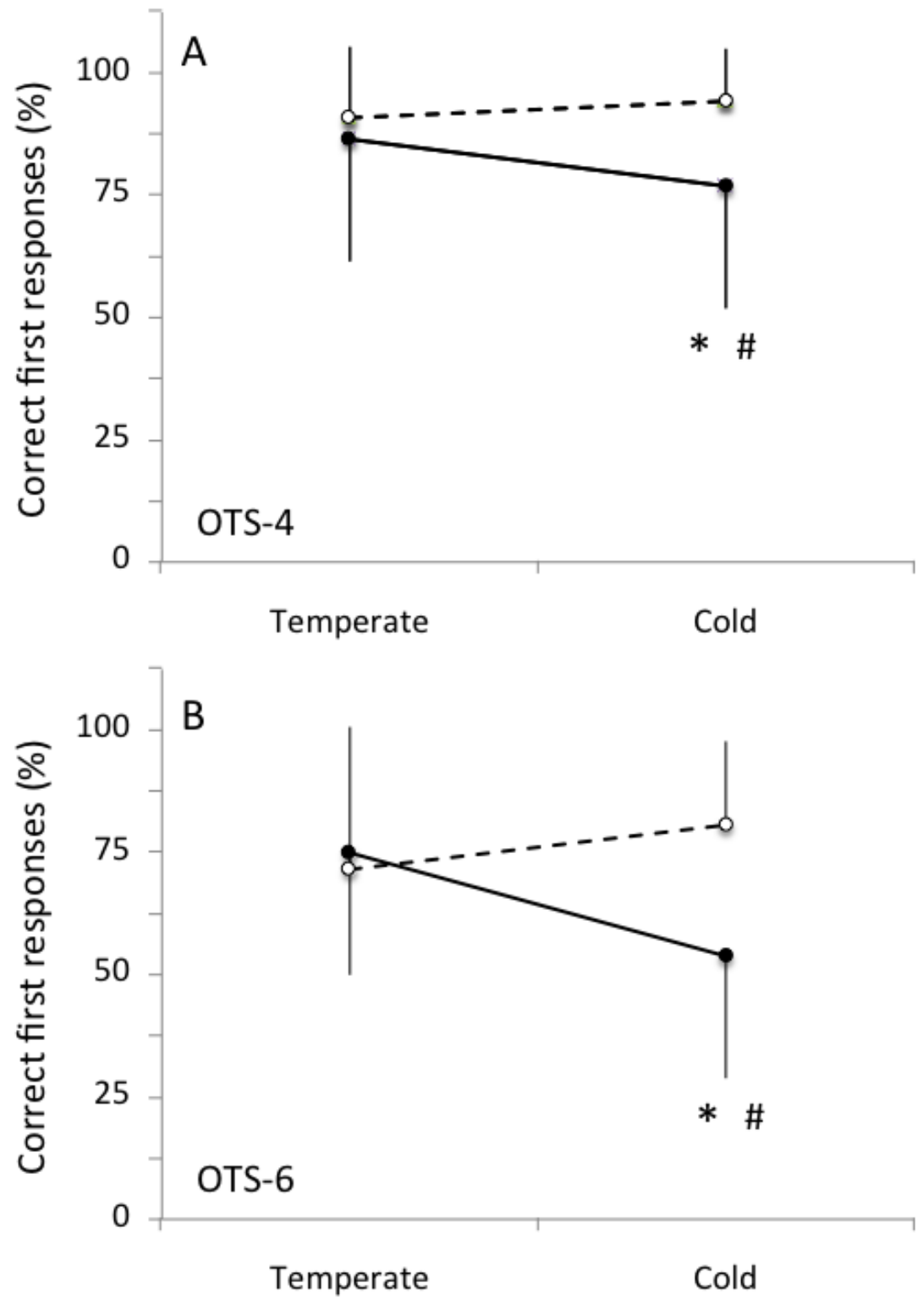

Figure 2. Cognitive performance. Percentage of successful trial on the first attempt over a total of 4 trial for a planning task requiring 4 (OTS-4) or 6 (OTS-6) movement in cold $\left(8^{\circ} \mathrm{C}\right)$ and temperate $\left(24^{\circ} \mathrm{C}\right)$ environments in elite skiers (dashed bars) and control participants (plain bars). \# difference between groups, $*$ difference between condition, $\mathrm{p}<0.05$. Value in mean $\pm \mathrm{SD}$. 
OTS-4
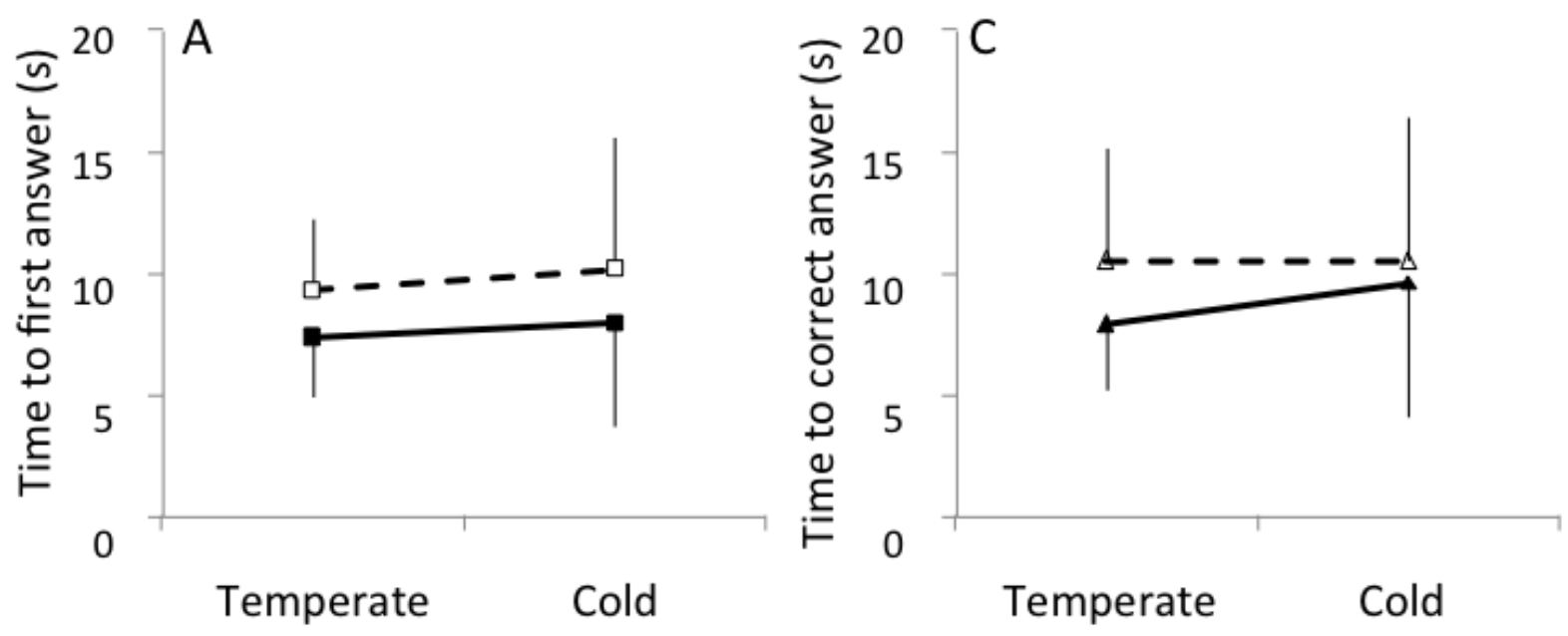

OTS-6
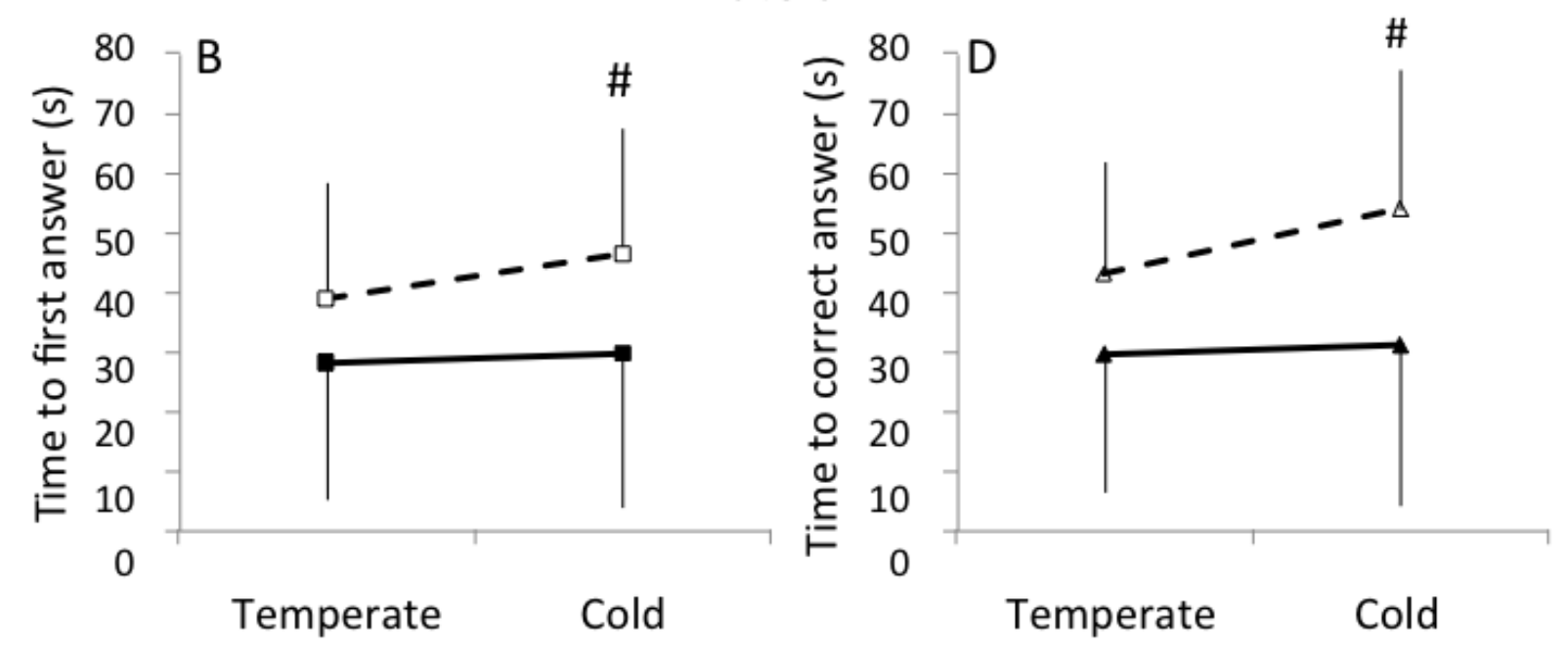

Figure 3. Response times during cognitive testing. Time to first answer (A, B) and correct answer (C, D) for a planning task requiring 4 (OTS-4; A, C) or 6 (OTS-6; B, D) movement in cold $\left(8^{\circ} \mathrm{C}\right)$ and temperate $\left(24^{\circ} \mathrm{C}\right)$ environments in elite skiers (dashed bars) and control participants (plain bars). \# difference between groups, $\mathrm{p}<0.05$. Value in mean $\pm \mathrm{SD}$. 
"Effect of Cold on Proprioception and Cognitive Function in Elite Alpine Skiers" by Racinais S et al. International Journal of Sports Physiology and Performance

(C) 2016 Human Kinetics, Inc.

Table 1. Physiological and subjective responses to cold exposure

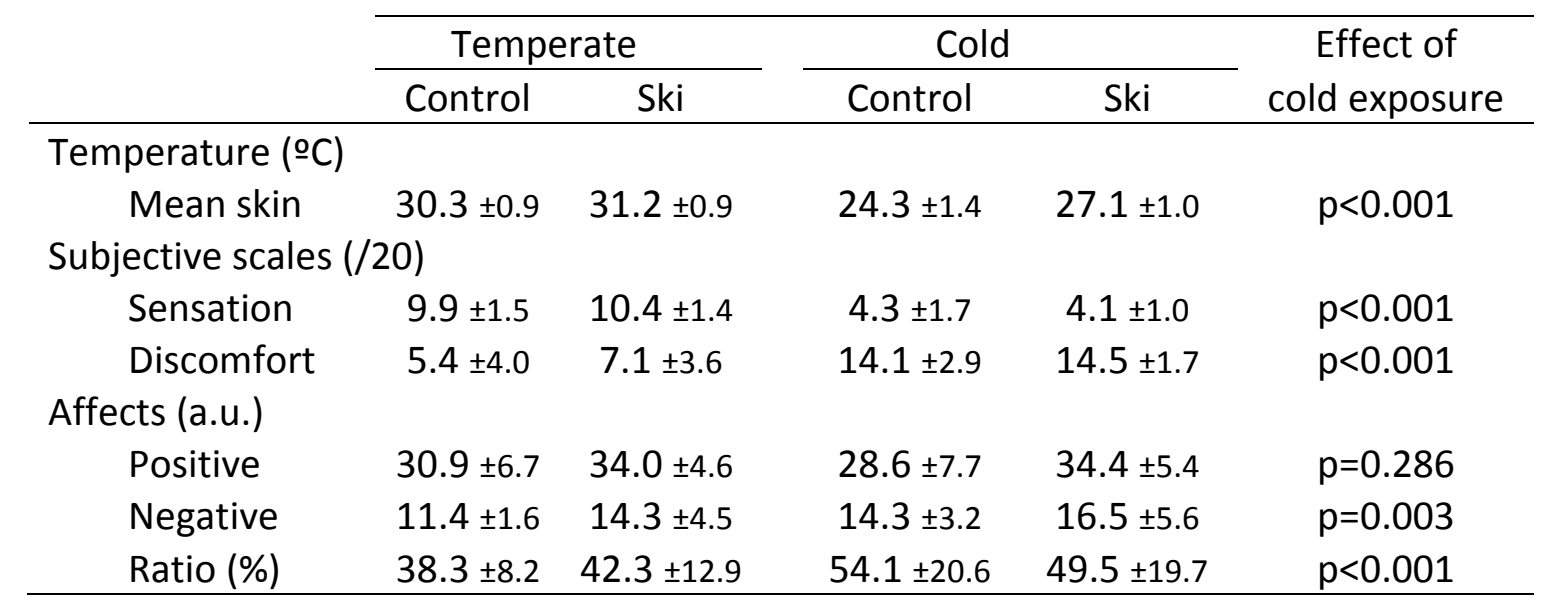

